\title{
BMJ Open Incidence and risk factors for type 2 diabetes mellitus in transitional Thailand: results from the Thai cohort study
}

\author{
Keren Papier, ${ }^{1,2}$ Susan Jordan, ${ }^{2}$ Catherine D‘Este, ${ }^{1}$ Chris Bain, ${ }^{1,2}$ \\ Janya Peungson, ${ }^{3}$ Cathy Banwell, ${ }^{1}$ Vasoontara Yiengprugsawan, ${ }^{1,4}$ \\ Sam-ang Seubsman, ${ }^{3}$ Adrian Sleigh ${ }^{1}$
}

To cite: Papier K, Jordan S, D‘Este C, et al. Incidence and risk factors for type 2 diabetes mellitus in transitional Thailand: results from the

Thai cohort study. BMJ Open 2016;6:e014102.

doi:10.1136/bmjopen-2016014102

- Prepublication history and additional material is available. To view please visit the journal (http://dx.doi.org/ 10.1136/bmjopen-2016014102).

Received 31 August 2016 Revised 2 November 2016 Accepted 16 November 2016

CrossMark

For numbered affiliations see end of article.

Correspondence to Ms Keren Papier; Keren.Papier@anu.edu.au

\section{ABSTRACT}

Background: Type 2 diabetes mellitus (T2DM) is increasingly prevalent in countries undergoing rapid development, including Thailand. We assessed T2DM incidence over an 8-year period in a nationwide cohort of Thai adults.

Methods: Thai Cohort Study participants were surveyed in 2005, 2009 and 2013. The analysed cohort members were aged (15-88), did not have diabetes in 2005 and were followed up by questionnaire in 2013 $(\mathrm{n}=39$ 507). T2DM was ascertained using self-report, which has been validated using physician interviews. We calculated the 8-year cumulative incidence of T2DM. Multivariable logistic regression assessed associations between potential risk factors and T2DM incidence.

Results: 8-year cumulative incidence of T2DM (2005 to 2013) was 177 per $10000(95 \% \mathrm{Cl} 164$ to 190$)$. Crude and age-standardised cumulative incidences of T2DM by sex were 249 per 10000 (95\% Cl 226 to 272) and 222 per 10000 (95\% Cl 219 to 225) for men; and 119 per 10000 (95\% Cl 105 to 133) and 96 per 10000 (95\% Cl 94 to 98$)$ for women, respectively. T2DM increased significantly for both sexes with increasing age and body mass index (BMI) ( $p$ trend $<0.001$ for both). Residence in an urban area as a child associated with T2DM among men and women (OR=1.4, 95\% Cl 1.1 to 1.7 and $\mathrm{OR}=1.4,95 \% \mathrm{Cl} 1.01$ to 1.79 ); this was no longer statistically significant after adjusting for BMI. Among men, smoking ( $\mathrm{OR}=1.7,95 \% \mathrm{Cl} 1.3$ to 2.2) and alcohol intake (OR=1.8, $95 \% \mathrm{Cl} 1.1$ to 3.0) were associated with T2DM.

Conclusions: This study found that the sociodemographic and lifestyle changes that have accompanied Thailand's economic development are associated with T2DM risk in a large cohort of Thai adults. Our findings highlight the need to address these transitions to prevent a further increase in the national incidence of T2DM, particularly among Thai men.

\section{BACKGROUND}

Rapid economic development accompanied by environmental, social and behavioural

\section{Strengths and limitations of this study}

- This nationwide study is the largest longitudinal study of health-risk factors and diabetes risk in Thailand.

- The participants in our cohort reflect Thais well socioeconomically and geographically.

- All diabetes diagnoses rely on self-report.

- An issue in this study is the loss to follow-up of cohort participants over the 8-year period.

change occurred in many low and middle-income countries (LMICs) over the past few decades. Concomitant shifts in behaviours ${ }^{1}$ led to a health-risk transition, including epidemiological and health transitions, ${ }^{2}{ }^{3}$ with falling infectious diseases, reduced childhood mortality and increases in noncommunicable diseases such as type 2 diabetes mellitus (T2DM). ${ }^{4}$

Thailand is one such country with rapidly emerging T2DM. Its prevalence among adults has risen from $2.3 \%$ in $1991^{5}$ to $8.0 \%$ in $2015 .{ }^{6}$ Over 4 million Thai adults live with diabetes, making it the top cause of disability-adjusted life years lost for Thai women and the seventh cause for men. ${ }^{7}$ T2DM is also an economic burden; in 2008 average annual cost per patient was US\$ 881 $-21 \%$ of per capita gross domestic product. ${ }^{8}$

Causes of the diabetes epidemic in industrialised countries are reasonably well established, ${ }^{9-11}$ but much less is known in LMICs such as Thailand. In developed countries, T2DM is inversely related with income and education while the opposite is usually noted when diabetes first emerges in LMICs. ${ }^{12} 13$ How environmental, social and behavioural changes are affecting T2DM risk among Thais is not known. The first three studies that reported on diabetes in Thailand were 
limited in size (under 7000 participants), location (Bangkok) or occupation (office workers, university employees or Electric Generation Authority plant workers). ${ }^{14-16}$ Despite the restrictions, these studies produced new knowledge for Thailand on downstream (eg, age and body mass index (BMI)) risk factors for T2DM. Incidence rates were estimated but upstream risks (geographical, socioeconomic) were not reported and the studies could not investigate beyond Bangkok. Our study was designed to fill this gap, is not geographically or occupationally restricted and is large in size. Accordingly, information emerging has application to the wide population of Thailand and as well as most of Southeast Asia.

Here we report an 8-year prospective cohort study providing nationwide data on incidence of T2DM and its risk factors. This information should help identify prevention targets and reveal the current state of the health transition in Thai adults.

\section{METHODS}

Study population

The Thai Cohort Study (TCS) is a longitudinal study of distance learning Open University students. It was established to investigate how rapid socioeconomic development is affecting health behaviours and outcomes in Thailand-the health-risk transition. ${ }^{3}$ In 2005, a 20-page questionnaire was mailed to all 200000 students enrolled at Sukothai Thammithirat Open University. These students are generally adults of modest means using education for self-improvement; they are embedded in their communities nationwide and are expected to experience the health-risk transition ahead of their fellow Thais. The questionnaires were self-completed in 2005, 2009 and 2013. Items included socioeconomic, demographic, cultural and lifestyle characteristics, health-risk behaviours and self-reported health outcomes including diabetes.

\section{Ascertainment of diabetes status}

At each wave of data collection, participants were classified as having diabetes if they responded positively to the question ('Have you ever received a confirmed diagnosis from a doctor that you definitely have diabetes?'). We used telephone interviews undertaken by a practicing Thai physician to confirm the validity of a large proportion of questionnaire-reported doctor-diagnosed T2DM and transient diabetes. Type 2 diabetes status was determined by the physician based on the participants' answers to a standard interview protocol. The protocol was developed following the American Diabetes Association's classification guidelines for diagnosing diabetes. ${ }^{17}$ The protocol included questions probing about blood sugar testing and cut-off criteria used for diagnosis, treatment and names of medications prescribed by the physician and about frequency of medical visits and the types of tests taken during medical visits. The validation study of these self-reported cases indicated high accuracy $(78 \%)$, particularly among those $(n=148)$ who reported doctor-diagnosed diabetes in 2009 and $2013(96 \%)$ (Unpublished data).

\section{Eligibility}

Participants were included in the study if they reported that they did not have diabetes at baseline in 2005 and if they provided diabetes status in the 2013 questionnaire. Excluded were those reporting diabetes at baseline (prevalent cases), and those reporting diabetes in 2009 and then reporting no diabetes in 2013 (mostly women with gestational diabetes).

\section{Assessment of risk factors}

We assessed health, lifestyle and sociodemographic variables reported at baseline as potential risk factors for incident T2DM. The sociodemographic information included age, personal monthly income, highest education level and area of residence in childhood (urban or rural). Health and lifestyle information included fruit, vegetable, tobacco and alcohol consumption and weight and height. ${ }^{18}$ For BMI, we divided weight in kilograms by height in $\mathrm{m}^{2}$ and categorised as recommended for Asian populations. ${ }^{19}$

Incidental exercise was measured by frequency of 'housework or gardening'. 'Leisure physical activity' scored as adjusted number of sessions per week of strenuous, moderate or mild exercise (' $2 \times$ strenuous +moderate+mild+walking' exercise sessions) $;^{20} \quad 21$ this weighted score was then categorised by sessions per week (none, $1-7,8-14,15$ or more).

\section{Statistical analysis}

\section{Cumulative incidence}

We calculated 8-year cumulative incidences of T2DM. Denominators included all participants who recorded no diabetes in 2005 and reported their diabetes status in 2013; numerators included those reporting having T2DM in 2013. Eight-year cumulative incidences were also stratified by age and sex. ${ }^{5}$ We also age-standardised the sex-specific rates to the WHO reference population for the year $2000 .^{22}$ We estimated age of onset as baseline age plus 2 years for those reporting T2DM in 2009 and baseline age plus 6 years for those reporting T2DM in 2013.

\section{Risk factors for incident T2DM}

We classified risk factors to be upstream (geographical, socioeconomic) or downstream (biomedical, personal). These different levels are important for designing the type of public health intervention. With T2DM as the outcome (yes/no), we used logistic regression to estimate ORs and $95 \%$ CIs for baseline risk factors.

Women in the cohort were, on average, younger than the men with $51 \%$ of women aged $<30$ at baseline versus $36 \%$ of men. Owing to the different age distributions and the potential for different associations by sex, all 
analyses were stratified by sex and adjusted for age. We used three models of increasing complexity. This enabled us to assess risk factors for T2DM with and without the impact of BMI, an important risk factor for T2DM. ${ }^{23}$ The first model (Model 1) had eight variants. One variant included age alone; the other seven variants each included one other risk factor of interest. The second model (Model 2) included all of the risk factors except for BMI. The third model (Model 3) included all risk factors and BMI. All analyses were carried out using Stata (V.13.0). A two-sided significance level of 0.05 was used.

\section{Sensitivity analysis}

We undertook two sensitivity analyses. For the first sensitivity analysis, we calculated the 4-year incidence of T2DM within each 4-year follow-up (2005 to 2009; 2009 to 2013) to determine whether the cumulative incidence estimates derived from these two 4-year periods were consistent with findings from the 8-year period (2005 to 2013).

In the second sensitivity analysis, we examined risk factors associated with T2DM incidence in the first 4 years (2005 to 2009) for four different subgroups: (1) everyone reporting incident T2DM in 2009; (2) excluding those reporting diabetes in 2009 but not in 2013; (3) excluding those lost to follow-up in 2013 and (4) only including those reporting T2DM in 2009 and 2013. Risk factor patterns for the four subgroups were compared with patterns for the 8-year results, assessing the effect of selection and information bias (attrition and misclassification).

Informed written consent was obtained from all participants. All data were de-identified before analysis.

\section{RESULTS}

\section{Participants}

The study population included TCS members who had been followed from 2005 to 2013, excluding 902 who reported diabetes at baseline and 167 who reported having diabetes in 2009 and not having diabetes in 2013, most being young women with transient (gestational) diabetes. Of the 39507 , the remaining cohort members initially at risk-698 reported being diagnosed with T2DM (figure 1).

The median (first and third quartiles) for age in years at baseline were $38(32,44)$ for those reporting T2DM and $31(26,37)$ for those who did not.

\section{Cumulative incidence of T2DM from 2005 to 2013}

Between 2005 and 2013, the overall T2DM cumulative incidence was 177 per 10000 (95\% CI 164 to 190). Corresponding crude and age-standardised cumulative incidences by sex were respectively: for the 17607 men, 249 per 10000 (95\% CI 226 to 272) and 222 per 10000 (95\% CI 219 to 225); for the 21900 women, 119 per 10000 (95\% CI 105 to 133) and 96 per 10000 (95\% CI
94 to 98 ). Figure 2 shows the age-sex-specific cumulative incidences of T2DM between 2005 and 2013. The incidence rose with age for both sexes, almost exponentially for men from age 50 .

\section{Risk factors for incident T2DM \\ Upstream risk factors}

For men, high income ( $>20000$ Baht per month) compared with low income (10000 Baht per month or less) and tertiary education relative to high school or less education statistically associated with T2DM in the models adjusted for age (Model 1) but not in the models adjusted for other risk factors (Models 2 and 3). There was no statistically significant association between T2DM and either income or education for women. For both sexes, there was a modest relationship with having lived in an urban area between the ages of 10 and 12 in Models 1 and 2 (Model 2:men OR=1.4, (95\% CI 1.1 to $1.7)$; women $\mathrm{OR}=1.4$, (95\% CI 1.01 to 1.79$)$ ), but after adjustment for BMI (Model 3), the magnitude of the effect estimates for urban residence approached unity, falling by $14 \%$ for men and $21 \%$ for women and were no longer statistically significant (table 1 ).

\section{Downstream risk factors}

Infrequent gardening or housework associated with significantly increased odds of T2DM for men in Model 1 only. In Model 2, for men and women, age was associated with T2DM ( $\geq 50$ years: OR=9.0, (95\% CI 5.8 to 14.0 ) and $\mathrm{OR}=9.9$, (95\% CI 5.2 to 19.0$)$, respectively). Obesity $\left(\mathrm{BMI} \geq 30.0 \mathrm{~kg} / \mathrm{m}^{2}\right)$ was associated with significantly increased odds of T2DM incidence in men $(\mathrm{OR}=23.1,95 \%$ CI 16.1 to 33.0) and women $(\mathrm{OR}=28.5$, 95\% CI 18.7 to 43.4 ), respectively (Model 3). Among men, regular alcohol intake $(\mathrm{OR}=1.8,(95 \%$ CI 1.1 to $3.0)$ ) and current smoking (OR=1.7, (95\% CI 1.3 to 2.2)) also associated with increased T2DM risk (Model $3)$. Addition of BMI to the models substantially attenuated OR estimates for age $(32 \%$ for men, $48 \%$ for women aged $\geq 50$ years) but had little influence on ORs for smoking, or alcohol (table 1).

\section{Sensitivity analysis}

Incidence across the two 4-year periods was stable-104 per 10000 (95\% CI 96 to 112) (2005 to 2009) and 105 per 10000 (95\% CI 95 to 115 ) (2009 to 2013). The cumulative incidence of T2DM per 10000 in men was approximately double that in women across the two 4-year periods (period 1:146 vs 70; period 2:146 vs 72). The findings from the two 4-year periods are consistent with those from the 8-year period (see online supplement 1).

The 2005 to 2009 4-year risk factor effect estimates were similar across the different subgroups (see Methods). An example from findings for men is shown in figure 3. Neither attrition nor accuracy of T2DM selfreport had an impact on the 8-year risk analyses. 


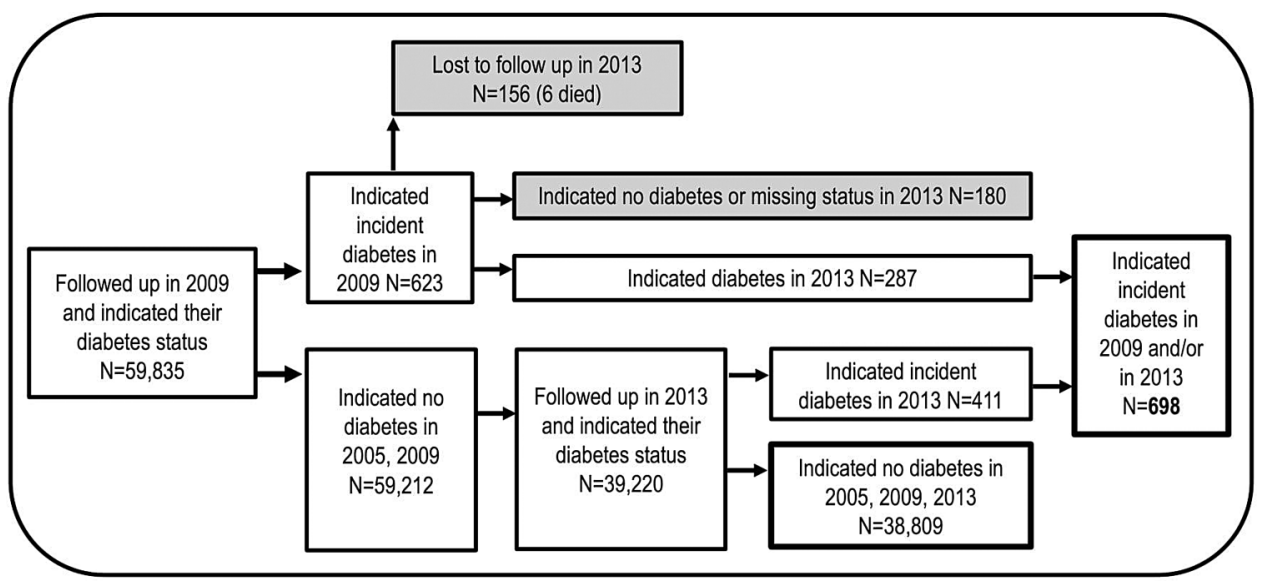

Figure 1 Selection of study participants from the Thai Cohort Study. *Participants were selected based on self-reported diabetes status in 2005 and available follow-up data in 2009 and 2013. The self-reported positives in 2005 were excluded $(n=902)$ as were those missing in 2009 and those with a missing diabetes status in their questionnaires. Eight-year cumulative incidence was calculated with the 39507 remaining participants in 2013.

\section{DISCUSSION}

We assessed factors associated with incidence of T2DM over 8 years in a nationwide cohort of young and middle-aged Thai adults. The 8-year cumulative incidence of T2DM between 2005 and 2013 was 177 per 10000 (95\% CI 164 to 190). T2DM incidence was higher among men (249 per 10 000; 95\% CI 226 to 272) than women (119 per 10000 ; $95 \%$ CI 105 to 133 ). For both sexes, factors most strongly associated with odds of developing T2DM were increasing age and higher BMI. Living in an urban area during childhood, smoking and alcohol are associated with increased risk of T2DM among men. However, most upstream associations attenuated when BMI was added to models. Tertiary education was associated with a small decrease in T2DM risk among women but this was not statistically significant.

There are limitations when interpreting these findings. All diabetes diagnoses used self-report so there may be case classification error. However, a validation study of self-reported diabetes conducted among TCS

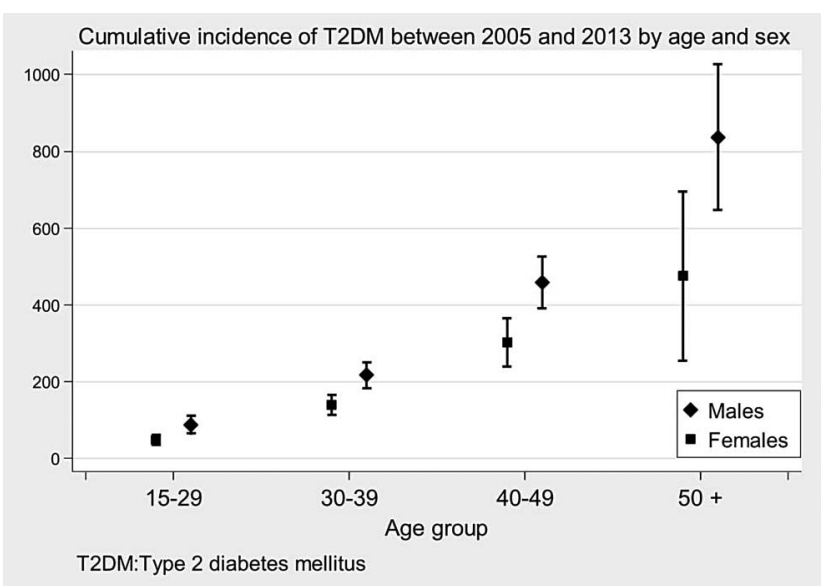

Figure 2 Cumulative incidence of T2DM between 2005 and 2013 by age and sex. participants indicated that accuracy of T2DM self-report was high. Also an issue is loss to follow-up. Overall, about $50 \%$ of the baseline cohort was retained after 8 years. A similar retention rate was noted for all values of sex, fruit, vegetable and alcohol intake and area of residence indicating that attrition for these variables should not be a concern. ${ }^{24}$ However, differential attrition was noted for the youngest age group, those underweight and those with the lowest income or lowest education and those who reported smoking in 2005. Differential attrition of participants who smoke might lead to an underestimation of T2DM incidence but differential retention of participants who are older and have a larger body size might lead to an overestimation of T2DM incidence. However, results in the first 4 years (70\% of baseline cohort) showed similar results to the total 8 years giving us confidence in generalisable findings.

This study has several strengths, including size, nationwide coverage and prospective longitudinal design. However, compared with the Thai population our cohort is younger, has higher levels of education and includes a higher proportion of adults living in metropolitan Bangkok. ${ }^{3}$ Incidence estimated by our study may be higher or lower than in the general population but the age-specific rates we report are expected to be generalisable, at least for educated groups. Furthermore, the participants in this cohort are ideal for studying the effects of sociodemographic change on T2DM risk since they are Thais of modest means, embedded in geographically dispersed communities across the nation and self-improving via education. Our population has been most informative and clearly represents well the large segment of the Thai population now entering the transition through a modern set of health concerns having undergone great changes in the environment, diet and lifestyle. ${ }^{3}$ We expect them to undergo the 'health-risk transition' ahead of fellow Thais. ${ }^{25} 26$ 
Table 1 Associations between baseline characteristics and 8-year cumulative incidence of diabetes (2005 to 2013), Thai Cohort Study

\begin{tabular}{|c|c|c|c|c|c|c|c|c|}
\hline \multirow[b]{2}{*}{$\begin{array}{l}\text { Baseline } \\
\text { characteristics }\end{array}$} & \multicolumn{2}{|c|}{ Diabetes status in 2013} & \multicolumn{3}{|c|}{ Men $\mathrm{N}=17607$ * OR (95\% Cl) } & \multicolumn{3}{|c|}{ Women $\mathrm{N}=21900$ * OR $(95 \% \mathrm{Cl})$} \\
\hline & $\begin{array}{l}\text { Incident } \\
\text { cases }\end{array}$ & $\begin{array}{l}\text { Total at } \\
\text { risk }\end{array}$ & Model 1 & Model 2 & Model 3 & Model 1 & Model 2 & Model 3 \\
\hline Age & n (\%) & $\mathrm{N}=39507^{*}$ & & & & & & \\
\hline $15-29$ & $108(1.0)$ & 17447 & 1 & 1 & 1 & 1 & 1 & 1 \\
\hline 30-39 & $252(2.0)$ & 14383 & 2.5 (1.8 to 3.4$)$ & $2.4(1.7$ to 3.4$)$ & 2.0 (1.4 to 2.8$)$ & $2.9(2.1$ to 4.1$)$ & $2.8(1.9$ to 4.1$)$ & 2.1 (1.4 to 3.0$)$ \\
\hline $40-49$ & $253(4.0)$ & 6506 & $5.4(4.0$ to 7.4$)$ & 4.7 (3.3 to 6.8$)$ & 3.4 (2.3 to 5.0$)$ & 6.5 (4.6 to 9.2$)$ & 6.3 (4.1 to 9.6$)$ & 3.8 (2.4 to 5.9$)$ \\
\hline 50 and over & $85(7.0)$ & 1171 & $10.4(7.2$ to 14.9$)$ & 9.0 (5.8 to 14.0$)$ & 6.1 (3.9 to 9.7$)$ & $10.4(6.0$ to 18.2$)$ & $9.9(5.2$ to 19.0$)$ & $5.1(2.6$ to 10.0$)$ \\
\hline \multicolumn{9}{|l|}{ Income(Baht/month) } \\
\hline$<10000$ & $267(1.0)$ & 22926 & 1 & 1 & 1 & 1 & 1 & 1 \\
\hline$\overline{10001-20000}$ & $227(2.0)$ & 10928 & $1.1(0.9$ to 1.4$)$ & $1.0(0.7$ to 1.2$)$ & $0.9(0.7$ to 1.1$)$ & $1.0(0.7$ to 1.3$)$ & $1.0(0.7$ to 1.4$)$ & $1.0(0.7$ to 1.5$)$ \\
\hline$>20001$ & $188(4.0)$ & 4907 & $1.3(1.00$ to 1.72$)$ & $1.1(0.8$ to 1.5$)$ & $1.1(0.8$ to 1.4$)$ & $1.0(0.7$ to 1.5$)$ & $0.9(0.6$ to 1.4$)$ & $1.1(0.7$ to 1.7$)$ \\
\hline \multicolumn{9}{|l|}{ Education } \\
\hline High school or less & $298(2.0)$ & 17084 & 1 & 1 & 1 & 1 & 1 & 1 \\
\hline Tertiary education & $396(2.0)$ & 22328 & $1.2(1.00$ to 1.47$)$ & $1.1(0.9$ to 1.4$)$ & 1.1 (0.9 to 1.3$)$ & $1.0(0.8$ to 1.3$)$ & 0.9 (0.7 to 1.2$)$ & 0.9 (0.7 to 1.2$)$ \\
\hline \multicolumn{9}{|c|}{ Childhood area of residence } \\
\hline Countryside (rural) & $448(2.0)$ & 29248 & 1 & 1 & 1 & 1 & 1 & 1 \\
\hline City/town (urban) & $237(2.0)$ & 9891 & 1.5 (1.2 to 1.9$)$ & $1.4(1.1$ to 1.7$)$ & $1.2(0.9$ to 1.5$)$ & $1.3(1.0$ to 1.7$)$ & $1.4(1.01$ to 1.79$)$ & $1.1(0.8$ to 1.4$)$ \\
\hline \multicolumn{9}{|c|}{ BMI $\left(\mathrm{kg} / \mathrm{m}^{2}\right)$ (Asian cut points) } \\
\hline Underweight $\leq 18.49$ & $8(0.2)$ & 5183 & $0.8(0.3$ to 1.9$)$ & & 0.7 (0.3 to 2.0$)$ & $0.2(0.1$ to 0.7$)$ & & $0.2(0.1$ to 0.8$)$ \\
\hline Normal (18.5-22.9) & $123(1.0)$ & 20997 & 1 & & 1 & 1 & & 1 \\
\hline At risk (23.0-24.9) & $121(2.0)$ & 6381 & 1.8 (1.3 to 2.6$)$ & & 1.9 (1.4 to 2.8$)$ & 3.6 (2.4 to 5.3$)$ & & 3.7 (2.4 to 5.7 ) \\
\hline Obese I (25.00-29.9) & $294(5.0)$ & 5489 & 5.1 (3.9 to 6.8$)$ & & 5.4 (3.9 to 7.3$)$ & 10.1 (7.2 to 14.2$)$ & & $10.4(7.2$ to 15.0$)$ \\
\hline Obese II $(\geq 30.0)$ & $142(15.0)$ & 971 & 20.6 (14.7 to 28.9 ) & & $23.1(16.1$ to 33.0$)$ & 27.7 (18.8 to 40.8$)$ & & 28.5 (18.7 to 43.4$)$ \\
\hline \multicolumn{9}{|c|}{ Gardening or housework } \\
\hline Most days & $362(2.0)$ & 22010 & 1 & 1 & 1 & 1 & 1 & 1 \\
\hline 1-2 times/week & $176(2.0)$ & 10617 & 1.0 (0.8 to 1.3$)$ & $1.0(0.8$ to 1.3$)$ & 0.9 (0.7 to 1.2$)$ & $1.2(0.9$ to 1.6$)$ & $1.2(0.9$ to 1.7$)$ & $1.3(0.95$ to 1.80$)$ \\
\hline$\leq 3$ times a month & $148(2.0)$ & 6429 & 1.3 (1.0 to 1.7$)$ & 1.2 (0.9 to 1.5$)$ & $1.0(0.8$ to 1.3$)$ & $1.3(0.9$ to 1.8$)$ & $1.1(0.7$ to 1.7$)$ & $1.0(0.7$ to 1.6$)$ \\
\hline \multicolumn{9}{|l|}{ Smoking } \\
\hline Never smoked & $384(1.0)$ & 28632 & 1 & 1 & 1 & 1 & 1 & 1 \\
\hline Ex-smoker & $157(3.0)$ & 6128 & $1.1(0.9$ to 1.4$)$ & $1.1(0.8$ to 1.4$)$ & $1.0(0.7$ to 1.3$)$ & 0.7 (0.3 to 1.6$)$ & 0.8 (0.4 to 1.9$)$ & $0.6(0.2$ to 1.5$)$ \\
\hline Current smoker & $119(4.0)$ & 3173 & 1.9 (1.5 to 2.4$)$ & 1.7 (1.3 to 2.2$)$ & 1.7 (1.3 to 2.2$)$ & 2.1 (0.8 to 5.9$)$ & $1.7(0.5$ to 5.6$)$ & $1.1(0.3$ to 4.0$)$ \\
\hline \multicolumn{9}{|l|}{ Alcohol intake } \\
\hline Never & $152(1.0)$ & 10873 & 1 & 1 & 1 & 1 & 1 & 1 \\
\hline Used to drink (quit) & $71(2.0)$ & 3252 & 1.5 (0.97 to 2.42) & 1.5 (0.9 to 2.5$)$ & 1.7 (1.0 to 2.9 ) & $0.6(0.3$ to 1.1$)$ & 0.6 (0.3 to 1.2$)$ & 0.6 (0.3 to 1.2$)$ \\
\hline Occasional/social & $399(2.0)$ & 23049 & 1.4 (0.9 to 2.0$)$ & $1.4(0.9$ to 2.1$)$ & 1.5 (0.96 to 2.37$)$ & $0.9(0.7$ to 1.1$)$ & 0.9 (0.7 to 1.2$)$ & 0.9 (0.2 to 1.3$)$ \\
\hline Regular drinker & $68(4.0)$ & 1900 & $2.0(1.3$ to 3.1$)$ & 1.7 (1.00 to 2.77$)$ & $1.8(1.1$ to 3.0$)$ & 0.5 (0.1 to 3.9$)$ & 0.5 (0.1 to 3.9$)$ & 0.7 (0.1 to 5.2$)$ \\
\hline
\end{tabular}

Model 1, age adjusted; Model 2, age and all variables except BMI; Model 3, Model 2 with BMI

*Numbers may not add to total sample size due to missing responses for some characteristics. 


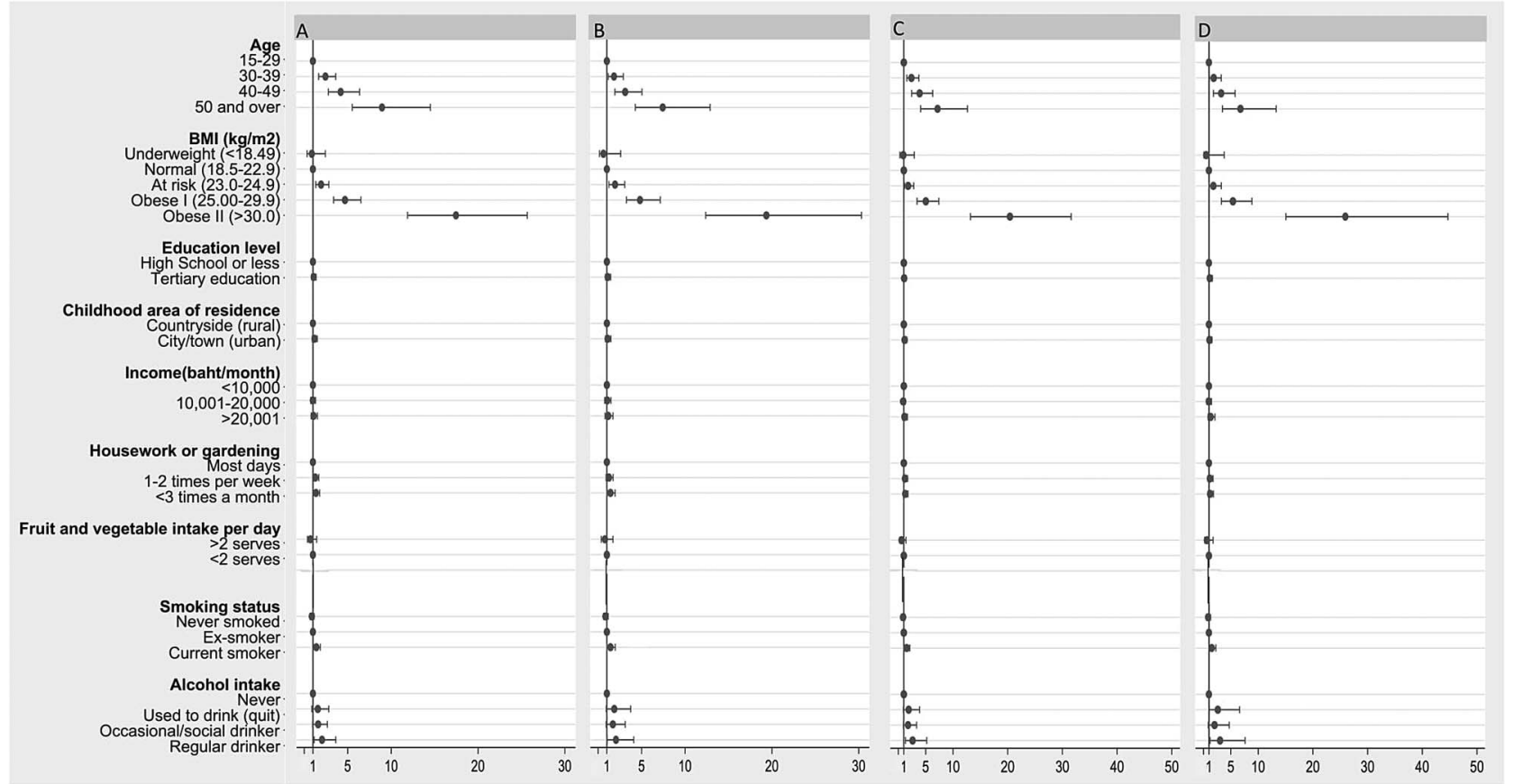

Figure 3 Sensitivity analysis comparing the ORs for incidence of diabetes between 2005 and 2009 according to diabetes reporting pattern in 2009 and 2013. Models were run for 4-year cumulative incidence between 2005 and 2009 among men, including the following participants. (A) Including all male participants followed up in 2009 ( $n=26885$ ). (B) Excluding the 107 male participants who reported having incident diabetes in 2009 but subsequently reported not having diabetes in $2013(n=26778)$.

(C) Excluding the 7949 male participants who were lost to follow-up in 2013 ( $n=18$ 936). (D) Excluding the 7949 male participants who were lost to follow-up in 2013 and the 107 male participants who reported having incident diabetes in 2009 but subsequently reported not having diabetes in 2013 ( $n=18$ 829).

The sex and age-specific cumulative incidence of T2DM between 2005 and 2013 was comparable with findings reported by previous Thai studies. ${ }^{14-16}$ Furthermore, our sex and age-specific estimates suggest that the incidence of T2DM among Thai cohort members is higher than Caucasian counterparts from North America ${ }^{27}$ and Europe ${ }^{28}$ similar to Bangladesh, ${ }^{29}$ and China $;{ }^{30}$ and lower than Pima Indians ${ }^{31}$ and Mauritians. ${ }^{32}$ However, direct comparisons are difficult due to differences in the case ascertainment, attrition and population sampling.

In the TCS, increasing age and BMI were the strongest risk factors for T2DM. Increasing age is a well-known risk for $\mathrm{T}_{2} \mathrm{DM}^{6}{ }^{19}$ and its effects were very apparent in this relatively young cohort. The high rates of T2DM incidence in our cohort members is consistent with findings from studies with Asian populations that have also shown that the risk of T2DM starts to increase at a relatively low age in Asian populations. ${ }^{33} 34$ The association with BMI is not surprising given that obesity is a wellestablished cause of diabetes. ${ }^{10}{ }^{35}$ Fat cells secrete hormones and adipokines that can increase the risk of diabetes through several pathways, including the increase in insulin resistance ${ }^{36}$ However, there are some notable differences between our findings and those from studies conducted in Caucasian populations. The incidence of T2DM in those with a BMI in the range of $23-24.9 \mathrm{~kg} / \mathrm{m}^{2}$ (healthy weight in Caucasians) ${ }^{37} 38$ was equivalent to the incidence rate of T2DM reported at higher BMI levels of $30.0 \mathrm{~kg} / \mathrm{m}^{2}$ in Caucasians. ${ }^{19} 38$ Our finding is consistent with findings from other Asian populations that have also shown that the risk of T2DM starts to increase at relatively low levels of BMI. ${ }^{33}$ T2DM and body size relations in Thai adults need further research.

Urbanisation accompanies socioeconomic growth in developing countries. ${ }^{39}$ We found that living in an urban area as a child increased risk of T2DM among men and women in models without adjustment for BMI. Previous reports from this cohort ${ }^{40}$ and other developing countries $^{12}$ shows urbanisation is associated with reduced physical activity, increased consumption of alcohol and highly processed food items and a higher BMI level. The attenuation of the association between urbanisation and T2DM risk that we observed after the addition of BMI to our model suggests that BMI has a major impact on the relationship between urbanisation and T2DM.

We found a 'developing country' pattern of increasing T2DM along with higher income and education for men (but not for women) in age-only adjusted models. The income and education effect were attenuated in the fully adjusted model. Other LMICs have shown that higher levels of education or socioeconomic status (SES) have a direct relationship with T2DM risk, whereas the opposite has been shown in developed western countries. ${ }^{13}$ However, once a country enters an advanced stage of economic development (equivalent to a gross national product per capita of around US\$ 
2500), the prevalence of obesity begins to rise predominantly in the group with the lowest SES and education level $^{13} \quad 4142$ and women are the first to manifest an inverse relationship between SES and obesity risk. ${ }^{41}$ Similar shifts appear to be occurring in the TCS cohort. ${ }^{43}$ Thus, our results suggest that women, at least in this cohort, are at a more advanced stage of the health transition. These findings highlight the need for public health interventions to target the risk factors for T2DM differently in men and women.

Men in this cohort are taking more health risks (smoking, regularly consuming alcohol and being less physically active) than the women ${ }^{44}$ and these risks link to T2DM. Public health efforts should preserve and encourage the low rates of alcohol consumption and smoking in Thai women to ensure that they do not adopt these new lifestyle behaviours, which lead to increased weight gain, insulin resistance and poor cardiovascular health. ${ }^{45}$ Concomitantly, the cessation of alcohol intake and smoking should be promoted among Thai men.

\section{CONCLUSION}

This study presents the 8-year cumulative incidence of T2DM between 2005 and 2013 and associated risk factors in a large cohort of Thai adults. We found that the incidence of T2DM was higher in men and that the lifestyle and sociodemographic changes that have accompanied Thailand's socioeconomic development are associated with T2DM risk. Thai men are likely to be in the middle stages of the health transition while women are more advanced. The focus of public health efforts should be on obesity, smoking and alcohol, particularly among men. The incidence of T2DM in Thailand is already high and many risks are converging especially obesity, ageing and physical inactivity. So we can expect T2DM will increase in importance rapidly over the next 1-2 decades and our data provide useful foresight regarding the growing impact of these changing risks.

\section{Author affiliations \\ ${ }^{1}$ National Centre for Epidemiology and Population Health (NCEPH) and Department of Global Health, Research School of Population Health, College of Medicine, Biology and Environment, The Australian National University, Canberra, Australia \\ ${ }^{2}$ Population Health Department, QIMR Berghofer Medical Research Institute, Brisbane, Australia \\ ${ }^{3}$ Thai Health-Risk Transition Study, School of Human Ecology, Sukhothai Thammathirat Open University, Nonthaburi, Thailand \\ ${ }^{4}$ Health and Wellbeing and Department of Global Health, Centre for Research on Ageing, Research School of Population Health, The Australian National University, Canberra, Australian Capital Territory, Australia}

\section{Twitter Follow Keren Papier @KerenPapier}

Acknowledgements We thank the team at Suhkkothai Thammathirat Open University (STOU) who assisted with student contact and the STOU students who are participating in the Thai Cohort Study.

Contributors KP devised the study, analysed all of the data and wrote the paper. SJ, CBain and AS assisted with the planning of the study and its required analyses, with the interpretation of the study findings and with the editing of all drafts. CD'E guided the analytical approach of this paper, supervised all analyses of the data and helped with the interpretation of the study findings. CBanwell, VY and JP assisted with the collection of the cohort data and with the editing of the manuscript. AS and SS conceived and developed the cohort. All authors approved the final manuscript.

Funding This study was supported by the International Collaborative Research Grants Scheme with joint grants from the Wellcome Trust UK (GR071587MA) and the Australian National Health and Medical Research Council (NHMRC, grant No.268055). It was also supported by a global health grant from the NHMRC (585426). SJ has a career development fellowship from the NHMRC. KP has an Australian Postgraduate Award from the Australian National University.

\section{Competing interests None declared.}

Ethics approval The Australian National University Human Research Ethics Committee (protocols 2004344 and 2009/570) and the Sukhothai Thammathirat Open University Research and Development Institute (protocol 0522/10).

Provenance and peer review Not commissioned; externally peer reviewed.

Data sharing statement No additional data are available.

Open Access This is an Open Access article distributed in accordance with the Creative Commons Attribution Non Commercial (CC BY-NC 4.0) license, which permits others to distribute, remix, adapt, build upon this work noncommercially, and license their derivative works on different terms, provided the original work is properly cited and the use is non-commercial. See: http:// creativecommons.org/licenses/by-nc/4.0/

\section{REFERENCES}

1. Ebrahim S, Smeeth L. Non-communicable diseases in low and middle-income countries: a priority or a distraction? Int J Epidemiol 2005;34:961-6.

2. Caldwell JC. Health transition: the cultural, social and behavioural determinants of health in the Third World. Soc Sci Med 1993;36:125-35.

3. Sleigh AC, Seubsman SA, Bain C, et al. Thai Cohort Study Team. Cohort profile: The Thai Cohort of 87134 Open University students. Int J Epidemiol 2008;37:266-72.

4. Yoon KH, Lee JH, Kim JW, et al. Epidemic obesity and type 2 diabetes in Asia. Lancet 2006;368:1681-8.

5. Aekplakorn W, Chariyalertsak S, Kessomboon P, et al., Thai National Health Examination Survey IV Study Group. Prevalence and management of diabetes and metabolic risk factors in Thai adults the Thai national health examination survey IV, 2009. Diabetes care 2011;34:1980-5.

6. International Diabetes Federation. IDF diabetes atlas. 7th edn. Brussels, Belgium: International Diabetes Federation, 2015

7. Bundhamcharoen $\mathrm{K}$, Odton $\mathrm{P}$, Phulkerd S, et al. Burden of disease in Thailand: changes in health gap between 1999 and 2004. BMC Public Health 2011;11:53.

8. Chatterjee S, Riewpaiboon A, Piyauthakit P, et al. Cost of diabetes and its complications in Thailand: a complete picture of economic burden. Health Soc Care Community 2011;19:289-98.

9. Williams ED, Tapp RJ, Magliano DJ, et al. Health behaviours, socioeconomic status and diabetes incidence: The Australian Diabetes Obesity and Lifestyle Study (AusDiab). Diabetologia 2010;53:2538-45

10. Geiss LS, Pan L, Cadwell B, et al. Changes in incidence of diabetes in US adults, 1997-2003. Am J Prev Med 2006;30:371-7.

11. Meisinger $C$, Thorand $B$, Schneider A, et al. Sex differences in risk factors for incident type 2 diabetes mellitus: the MONICA Augsburg cohort study. Arch Intern Med 2002;162:82-9.

12. Angkurawaranon $C$, Jiraporncharoen $\mathrm{W}$, Chenthanakij $\mathrm{B}$, et al. Urbanization and non-communicable disease in Southeast Asia: a review of current evidence. Public health 2014;128:886-95.

13. Agardh E, Allebeck P, Hallqvist J, et al. Type 2 diabetes incidence and socio-economic position: a systematic review and meta-analysis. Int J Epidemiol 2011;40:804-18.

14. Jiamjarasrangsi W, Aekplakorn W. Incidence and predictors of type 2 diabetes among professional and office workers in Bangkok, Thailand. J Med Assoc Thai 2005;88:1896-904.

15. Jiamjarasrangsi $\mathrm{W}$, Lohsoonthorn $\mathrm{V}$, Lertmaharit $\mathrm{S}$, et al. Incidence and predictors of abnormal fasting plasma glucose among the 
university hospital employees in Thailand. Diabetes Res Clin Pract 2008;79:343-9.

16. Aekplakorn W, Bunnag $P$, Woodward $M$, et al. A risk score for predicting incident diabetes in the Thai population. Diabetes care 2006;29:1872-7.

17. American Diabetes Association. Diagnosis and classification of diabetes mellitus. Diabetes Care 2010;33(Suppl 1):S62-9.

18. Lim LL, Seubsman SA, Sleigh A. Validity of self-reported weight, height, and body mass index among university students in Thailand: Implications for population studies of obesity in developing countries. Popul Health Metr 2009;7:15

19. Chiu M, Austin PC, Manuel DG, et al. Deriving ethnic-specific BM cutoff points for assessing diabetes risk. Diabetes Care 2011;34:1741-8.

20. Banks E, Lim L, Seubsman SA, et al. Relationship of obesity to physical activity, domestic activities, and sedentary behaviours: cross-sectional findings from a national cohort of over 70,000 Thai adults. BMC Public Health 2011;11:762.

21. Australian Institute of Health and Welfare. The active Australia survey: a guide and manual for implementation, analysis and reporting. Canberra: Australian Institute of Health and Welfare, 2003:1-55.

22. Ahmad OB, Boschi-Pinto C, Lopez AD, et al. Age standardization of rates: a new WHO standard. Geneva: World Health Organization, 2001.

23. Astrup A, Finer N. Redefining type 2 diabetes:'diabesity'or 'obesity dependent diabetes mellitus'? Obes Rev 2000;1:57-9.

24. Thinkhamrop K, Seubsman SA, Sleigh A, et al. Reasons for Non-Response to Mailed Questionnaires-Experience of a Large Cohort Study in Thailand. J Public Health Res 2012;4:73-87.

25. Thawornchaisit P, de Looze F, Reid CM, et al., Thai Cohort Study Team*. Health risk factors and the incidence of hypertension: 4-year prospective findings from a national cohort of 60569 Thai Open University students. BMJ Open 2013;3:e002826.

26. Yiengprugsawan V, Horta BL, Motta JV, et al. Body size dynamics in young adults: 8-year follow up of cohorts in Brazil and Thailand. Nutr Diabetes 2016;6:e219.

27. Lipscombe LL, Hux JE. Trends in diabetes prevalence, incidence, and mortality in Ontario, Canada 1995-2005: a population-based study. Lancet 2007;369:750-6.

28. Bonora E, Kiechl S, Willeit J, et al. Population-based incidence rates and risk factors for type 2 diabetes in white individuals: the Bruneck study. Diabetes 2004;53:1782-9.

29. Asghar S, Khan AK, Ali SM, et al. Incidence of diabetes in Asian-Indian subjects: a five year follow-up study from Bangladesh. Prim Care Diabetes 2011;5:117-24.

30. Chen R, Song Y, Hu Z, et al. Predictors of diabetes in older people in urban China. PloS one 2012;7:e50957.
31. Pavkov ME, Hanson RL, Knowler WC, et al. Changing patterns of type 2 diabetes incidence among Pima Indians. Diabetes care 2007;30:1758-63.

32. Söderberg S, Zimmet P, Tuomilehto J, et al. High incidence of type 2 diabetes and increasing conversion rates from impaired fasting glucose and impaired glucose tolerance to diabetes in Mauritius. J Intern Med 2004;256:37-47.

33. He L, Tuomilehto J, Qiao Q, et al. Impact of classical risk factors of type 2 diabetes among Asian Indian, Chinese and Japanese populations. Diabetes Metab 2015;41:401-9.

34. Nakagami T, Qiao Q, Carstensen B, et al., DECODE-DECODA Study Group. . Age, body mass index and type 2 diabetesassociations modified by ethnicity. Diabetologia 2003;46:1063-70.

35. Wang C, Li J, Xue H, et al. Type 2 diabetes mellitus incidence in Chinese: Contributions of overweight and obesity. Diabetes Res Clin Pract 2015;107:424-32.

36. Bray GA. Medical consequences of obesity. J Clin Endocrinol Metab 2004;89:2583-9.

37. Ma RC, Chan JC. Type 2 diabetes in East Asians: similarities and differences with populations in Europe and the United States. Ann N Y Acad Sci 2013;1281:64-91.

38. Stevens J, Truesdale KP, Katz EG, et al. Impact of body mass index on incident hypertension and diabetes in Chinese Asians, American Whites, and American Blacks The People's Republic of China Study and the Atherosclerosis Risk in Communities Study. Am J Epidemiol 2008;167:1365-74.

39. Popkin BM. Urbanization, lifestyle changes and the nutrition transition. World Dev 1999;27:1905-16.

40. L-Y Lim L, Kjellstrom T, Sleigh A, et al. Associations between urbanisation and components of the health-risk transition in Thailand. A descriptive study of 87,000 Thai adults. Global Health Action 2009;2. doi: 10.3402/gha.v52i0.1914

41. Monteiro CA, Moura EC, Conde WL, et al. Socioeconomic status and obesity in adult populations of developing countries: a review. Bull World Health Organ 2004;82:940-6.

42. Jones-Smith JC, Gordon-Larsen P, Siddiqi A, et al. Is the burden of overweight shifting to the poor across the globe? Time trends among women in 39 low-and middle-income countries (1991-2008). Int $J$ Obes (Lond) 2012;36:1114-20.

43. Seubsman S, Lim LL, Banwell C, et al. Socioeconomic status, sex and obesity in a large national cohort of 15-87-year-old open university students in Thailand. J Epidemiol 2010;20:13-20.

44. Wakabayashi M, McKetin R, Banwell C, et al., Thai Cohort Study Team. Alcohol consumption patterns in Thailand and their relationship with non-communicable disease. BMC Public Health 2015;15:1297

45. Hu FB, Manson JE, Stampfer MJ, et al. Diet, lifestyle, and the risk of type 2 diabetes mellitus in women. N Engl J Med 2001;345:790-7. 